\title{
Design and construction of multi-cylinder type liquid pis- ton Stirling engine
}

\author{
Prastowo Murti ${ }^{1,2, *}$, Akira Takizawa ${ }^{1}$, Eita Shoji ${ }^{1}$, and Tetsushi Biwa ${ }^{1}$ \\ ${ }^{1}$ Department of Mechanical Systems Engineering, Tohoku University, 6-6, Aramaki, Aoba-Ku, Sendai, \\ 980-8579, Japan \\ ${ }^{2}$ Department of Mechanical \& Industrial Engineering, Faculty of Engineering, Universitas Gadjah \\ Mada, Jl. Grafika No. 2, Yogyakarta 55281, Indonesia
}

\begin{abstract}
In a multi-cylinder type liquid piston Stirling engine (MCLPSE), liquid columns in U-shaped tubes play the role of solid pistons in a mechanical Stirling engine. Besides the straightforward structure, advantages of the MCLPSE are a relatively low operation temperature difference below $100 \mathrm{~K}$ and use of harmless working fluids of air and water. This study presents a mass spring model for the MCLPSE, from which we determine geometrical parameters of MCLPSE to achieve a target acoustic power production under a given temperature condition. The preliminary test results will be presented.
\end{abstract}

\section{Introduction}

An effort to eliminate solid piston in conventional Stirling engine has been successfully achieved by using thermally induced acoustic traveling waves in a looped tube [1]. The idea came from noticing that the phasing of pressure and velocity in traveling waves are essentially the same as that in the regenerator of the Stirling engine [2]. To enhance the engine's performance, Los Alamos National Laboratory researchers connected the branch resonator to the looped tube [3]. The resulting thermal efficiency was as high as 30\%, which is comparable with internal combustion engine $(25-40 \%)$. The improvement was attributable to the specific acoustic impedance $z$ (ratio of pressure $p$ and velocity $u$ of the acoustic gas oscillations) in the regenerator that was increased to as high as $30 \rho_{\text {gas }} c$, where $\rho_{\text {gas }}$ and $c$ denote the density and adiabatic speed of sound of the gas, respectively. Such a high specific acoustic impedance led to reduction of the viscous losses that are inevitably induced when gases oscillate in the narrow channel of regenerator. Therefore, a traveling wave phasing and a high specific acoustic impedance are the keys to increase the thermal efficiency of the thermoacoustic Stirling engine.

Use of liquid column oscillations in a U-shaped tube is the another method to eliminate the solid piston. C. West in the 1970's [4] illustrated a conceptual design of a liquid piston Stirling engine consisting of multiple unit sections, as shown in Fig. 1(a). Each unit section comprises of a U-tube filled with working liquid and gas. They are connected sequentially to form a ring. A regenerator and a pair of heat exchangers are contained in the gas-filled section on the top, as shown in Fig. 1(b). This type of engine can be called the multicylinder type of liquid piston Stirling engine (MCLPSE). The first operation of MCLPSE with

\footnotetext{
*e-mail: murti.prastowo.r2@dc.tohoku.ac.jp
} 


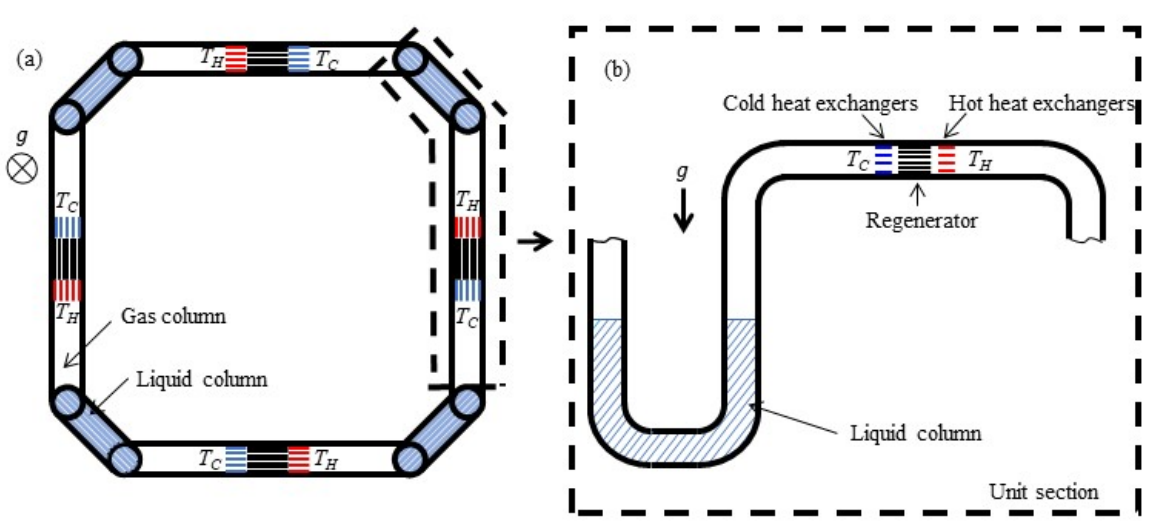

Figure 1. Top view of the MCLPSE (a) and a cross-sectional view of a unit section (b). The gravitational acceleration is denoted by $g$.

four unit sections was reported in 1983 by Martini [5]. He showed that the adjacent liquid columns oscillated with 90 degrees out of phase when the heating temperature was in a range of $170-200^{\circ} \mathrm{C}$. A recent study reported that an equivalent mechanical model of MCLPSE based on masses and springs predicted the natural oscillation frequency and specific acoustic impedance, which assured the traveling wave phasing in the regenerator and the adjustability of the specific acoustic impedance by the filling ratio of water [6]. Therefore, MCLSPE is also a candidate of a Stirling engine with simple structure and reasonable thermal efficiency.

This study presents the design and experimental validation of multi-cylinder type liquid piston Stirling engine based on the mass-spring model built in the previous paper [6]. In this study, we aim at producing $20 \mathrm{~W}$ of acoustic power generation with cold and hot end temperatures of $30^{\circ} \mathrm{C}$ and $140^{\circ} \mathrm{C}$, respectively, using air and water at atmospheric pressure and temperature as working fluids. The acoustic power generation means the power generated to sustain the oscillations, because the present engine had no external load. The experimental results were presented and compared with the calculation results.

\section{Design and optimization processes}

We determine the geometrical parameters of the engine, such as the unit section length $L$ and the filling ratio $\varepsilon$ of the liquid column length over $L$, as they are related to the production of acoustic power when the acceleration amplitude of the longitudinal oscillation of the liquid column $\alpha$ is limited by the Rayleigh-Taylor instability. The mass-springs model shown in Fig. 2, consists of point mass and two kinds of springs. The point mass $m=A \rho_{l} \varepsilon L$ represents the liquid column, coil spring as the gas column with spring constant $k_{a}=A \gamma P_{m} /[(1-\varepsilon) L]$, and leaf spring as gravitational force acting on the liquid column with spring constant $k_{g}=$ $2 A \rho_{l} g$, where $A$ denotes the cross-sectional area of tube, $P_{m}$ denotes mean pressure, $\gamma$ denotes specific heat ratio of the gas, $\rho_{l}$ denotes density of liquid, $g$ denotes gravitational acceleration. The number of mass represents number of unit sections $n$. In this study, we focus on the natural oscillation mode of the MCLPSE and ignored the damping effect of the liquid column. (For a numerical simulation result involving the viscosity and thermal conductivity of the working fluids, see the reference [7].) 
By solving the equations of motion under a periodic boundary condition, the natural angular frequency $\omega$ for the fundamental oscillation mode is given as

$$
\omega^{2}=\frac{k_{g}}{m}+\frac{k_{a}}{m}\left(1-\cos \frac{2 \pi}{n}\right),
$$

which can be rewritten as

$$
\omega^{2}=\frac{2 g}{\varepsilon L}+\frac{2 \gamma P_{m}}{\rho_{l} \varepsilon(1-\varepsilon) L^{2}}\left(1-\cos \frac{2 \pi}{n}\right) .
$$

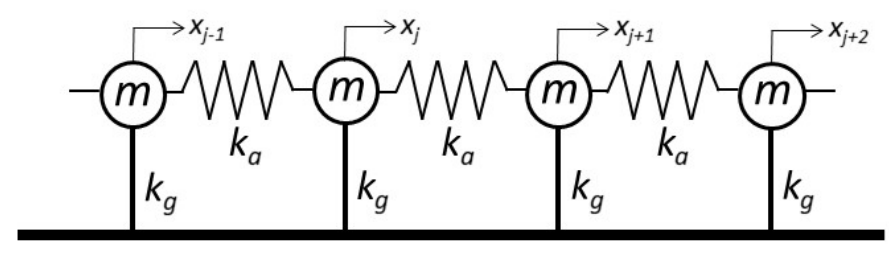

Figure 2. Mass-spring equivalent model [6]. Symbol $x_{j}$ denotes displacement of $j$-th mass from the equilibrium position.

The complex gas pressure $P_{j}$ is given by using the difference in the displacements of the adjacent liquid columns as

$$
P_{j}=\frac{k_{a}}{A}\left(X_{j}-X_{j+1}\right),
$$

where $X_{j}$ and $X_{j+1}$ are complex amplitudes for the displacement oscillations of $j$-th and $(j+1)$ th liquid columns. They are also given from the solution of the equations of motions by

$$
X_{j}=C \exp \left( \pm i \frac{2 \pi j}{n}\right)
$$

where $C$ is constant and $i$ is imaginary unit.

The complex gas velocity $u$ in the middle of the gas column is assumed by the average velocity of the liquid columns. It is expressed by

$$
u_{j}=\frac{i \omega}{2}\left(X_{j}+X_{j+1}\right)
$$

The specific acoustic impedance $z=P / u$ in the middle of gas column is given, independently of $j$, by

$$
\frac{z}{\rho_{\text {gas }} c}=\frac{2 c}{\omega(1-\varepsilon) L} \tan \frac{\pi}{n} .
$$

We assume that the displacement amplitude $|C|$ of liquid column is limited by the acceleration amplitude. Therefore, we assume $|C|=\alpha / \omega^{2}$, and hence, we have

$$
\left|u_{j}\right|=\frac{\alpha}{\omega} \cos \frac{\pi}{n} .
$$

The acoustic power $\left(W=\frac{A}{2} \operatorname{Re}[z]\left|u_{j}\right|^{2}\right)$ in the middle of the gas column is given by

$$
W=\frac{A \rho_{g a s} c^{2} \alpha^{2}}{2 \omega^{3}(1-\varepsilon) L} \sin \frac{2 \pi}{n} .
$$


Consider the regenerator placed in the middle of the gas column having end temperatures of $T_{H}$ and $T_{C}$. The acoustic power entering the regenerator is amplified with a power gain equal to a temperature ratio of $T_{H} / T_{C}$ [8]. Thus, the acoustic power $W_{\text {out }}$ leaving the regenerator is larger than the power $W_{i n}$ entering the regenerator. The acoustic power generation per regenerator is expressed by $\Delta W=\left(W_{\text {out }}-W_{\text {in }}\right)$, which is the result of the Stirling thermodynamic cycle in the regenerator. The acoustic power generation $\Delta W$ is expressed as follows

$$
\Delta W=2 W \frac{T_{H}-T_{C}}{T_{H}+T_{C}},
$$

where we assumed $W=\left(W_{\text {out }}+W_{\text {in }}\right) / 2$ and $W_{\text {out }} / W_{\text {in }}=T_{H} / T_{C}$. Therefore, the desired acoustic power generation each unit section can be obtained by specifying parameters in Eqs. 8 and 9.

The calculation starts by giving initial input parameters as follows; the diameter of the tube $D_{\text {tube }}=2 \sqrt{A / \pi}$ is $40 \mathrm{~mm}$, the number of unit sections $n$ is 4 , the working fluids are air and water at atmospheric pressure, the acceleration amplitude of the liquid piston $\alpha$ is $2 g$, and the regenerator ends temperature assumed $T_{C}=30^{\circ} \mathrm{C}$ and $T_{H}=140^{\circ} \mathrm{C}$. Figure (2) shows 3D plot of (a) $\Delta W$, (b) $f$, and (c) $z /\left(\rho_{\text {gas }} c\right.$ ) as functions of $L$ and $\varepsilon$. In Fig. 2(a), $\Delta W$ increases with $L$ but reaches maximum at approximately $\varepsilon=0.78$ for any $L$. $\Delta W$ increases with $L$ because $f$ decreases as shown in Fig. 2(b). The reduction of $f$ increases velocity amplitude through a factor of $\alpha / \omega$ in Eq. (7). When $f$ is viewed as a function of $\varepsilon$, it becomes the lowest when $\varepsilon=0.53$, although $\Delta W$ shows a peak as we stated above. The difference between $\varepsilon=0.78$ and $\varepsilon=0.53$ arises from the specific acoustic impedance shown in Fig. 2(c). The specific acoustic impedance $z /\left(\rho_{\text {gas }} c\right)$ maintains a constant value with any of $L$, whereas it increases with an increase of $\varepsilon$. Since $W$ is proportional to $z, \Delta W$ shows a peak with $\varepsilon=0.78$.

To achieve a total $\Delta W$ of $20 \mathrm{~W}$, each unit section should produce $\Delta W$ of $5 \mathrm{~W}$. As shown in Fig. 2(a), in this study, the filling ratio $\varepsilon$ of 0.58 and the length of the unit section $L$ of $3 \mathrm{~m}$ were chosen, although $\Delta W$ of $5 \mathrm{~W}$ can be generated with different combination values of $\varepsilon$ and $L$. At the same $\varepsilon$ and $L$ values, from Figs. 2(b) and 2(c), the oscillation frequency $f$ and specific acoustic impedance $z /\left(\rho_{\text {gas }} c\right)$ are $1.88 \mathrm{~Hz}$ and 46.59 , respectively. Moreover, the displacement amplitude of liquid $\left(X_{\text {liq }}=\alpha /(2 \pi f)^{2}\right)$ reached $0.14 \mathrm{~m}$ when the acceleration amplitude $\alpha$ reaches $2 g$ under the temperature conditions of $T_{C}=30^{\circ} \mathrm{C}$ and $T_{H}=140^{\circ} \mathrm{C}$.

\section{Experimental verification}

\subsection{Experimental setup}

The experimental setup of MCLPSE was built to verify the results of design results. The number of unit section $n$ was four, as shown in Fig 1 . Each unit section consisted of $D_{\text {tube }}$ $=40 \mathrm{~mm}$ with total length of the unit section $L=3 \mathrm{~m}$. The ratio of the liquid column length to the total unit section length was $\varepsilon=0.58$. The working fluids were air and water at atmospheric pressure and temperature. The regenerator was made of a cylindrical ceramic honeycomb catalyst support which had square pores with a side of $2 r_{h}=0.68 \mathrm{~mm}$ and length of $70 \mathrm{~mm}$. The hot heat exchanger was made of an $85 \mathrm{~mm}$ length of the cylinder block of copper with rows of holes drilled axially with a diameter of $3.5 \mathrm{~mm}$. The cold heat exchanger was made of many tubes arranged triangular with inner diameter of $3.5 \mathrm{~mm}$ and $100 \mathrm{~mm}$ in length placed inside the shell (body).

The hot heat exchanger was heated by an electric heater, whereas the cold heat exchanger was cooled by circulating water with a temperature of $23^{\circ} \mathrm{C}$. In order to facilitate visual observation of liquid column displacement $X_{\text {liq }}$ using high speed camera, the liquid column tube was made of transparent PVC tubing. Pressure oscillations in the gas column were 

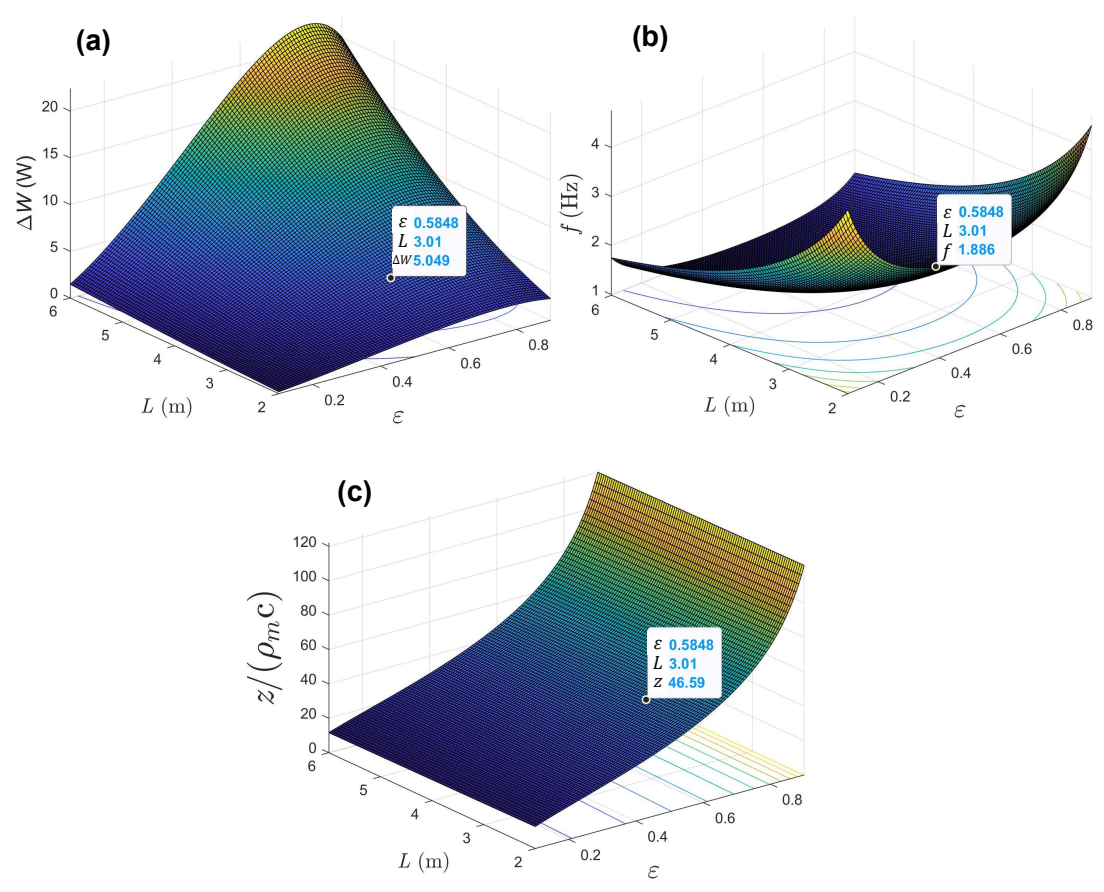

Figure 3. 3D plot of (a) $\Delta W$, (b) $f$, and (c) $z / \rho_{m} c$ as in the plane of $L$ and $\varepsilon$. The marked point represents the determined values of $L$ and $\varepsilon$, and the corresponding $\Delta W, f$, and $z / \rho_{m} c$.

monitored using pressure transducers. Two K-type thermocouples were placed at ends of the regenerators to measure the temperature $T_{H}$ and $T_{C}$. A cylindrical rod of ultra-highmolecular-weight polyethylene with a diameter of $38 \mathrm{~mm}$ and a length of $300 \mathrm{~mm}$ was used as a float to suppress the liquid surface instability [9].

\subsection{Experimental Results}

The onset temperature of the engine was determined through the measurement of quality factor $Q[10,11]$. Figure 4(a) shows the inverse of measured quality factor $1 / Q$ as a function of temperature difference $T_{H}-T_{C}$ between ends of the regenerator. It is found that $1 / Q$ decreased by increasing $T_{H}-T_{C}$. A decrease in $1 / Q$ indicated the reduction of energy dissipation associated with the oscillations of gas and liquid columns. The onset temperature difference of the engine was deduced to be $38^{\circ} \mathrm{C}$ when $1 / Q$ reaches zero, because we are able to say that the acoustic power generation in the regenerator overcomes the energy dissipation with this temperature difference. The onset temperature difference of $38^{\circ} \mathrm{C}$ is remarkably low. Therefore, the MCLPSE can be used as a simple energy conversion device that utilized any low-grade heat sources. In the steady oscillation states of the engine, we found that the oscillation frequency $f$ was $1.906 \mathrm{~Hz}$, and 90-degree phase difference between the oscillations of adjacent liquid columns.

Figure 4(b) shows the pressure and liquid displacement amplitudes as a function of $T_{H}-$ $T_{C}$. It can be seen that both pressure and liquid displacement amplitudes increased with $T_{H}-T_{C}$. The pressure amplitude $P$ of $21.93 \mathrm{kPa}$ was achieved when the $T_{H}-T_{C}$ was 
$116^{\circ} \mathrm{C}$ with $T_{H}=141^{\circ} \mathrm{C}$ and $T_{C}=25^{\circ} \mathrm{C}$. At the same condition, the liquid displacement amplitude $X_{l i q}$ was $0.142 \mathrm{~m}$. The acceleration amplitude $\left(\alpha=X_{l i q}(2 \pi f)^{2}\right)$ of $20.36 \mathrm{~m} / \mathrm{s}^{2}$ was obtained. As a result, the gas velocity amplitude $u$ in the middle of gas column was estimated to be $1.2 \mathrm{~m} / \mathrm{s}$ from Eq. (7). Therefore, the specific acoustic impedance $\left(z / \rho_{\text {gas }} c\right)$ at the same location was 45.32, which was consistent with the result of the mass-spring model. Using the gas pressure and velocity, we estimated the acoustic power in middle of regenerator $\left(W=\frac{A}{2} P u\right)$ and acoustic power generation $\Delta W$ through Eq. 9. It is obtained that $W=16.53$ $\mathrm{W}$; thus, the acoustic power generation $\Delta W$ in each regenerator was $5.35 \mathrm{~W}$. Therefore, the total acoustic power generation is $21.4 \mathrm{~W}$. The results of this study confirmed that MCLPSE can be designed easily. The next stage would be to combine the mass-spring model with DeltaEC $[12,13]$, in order to see if the desired acceleration amplitude is attained with a given temperature conditions of $T_{H}$ and $T_{C}$.
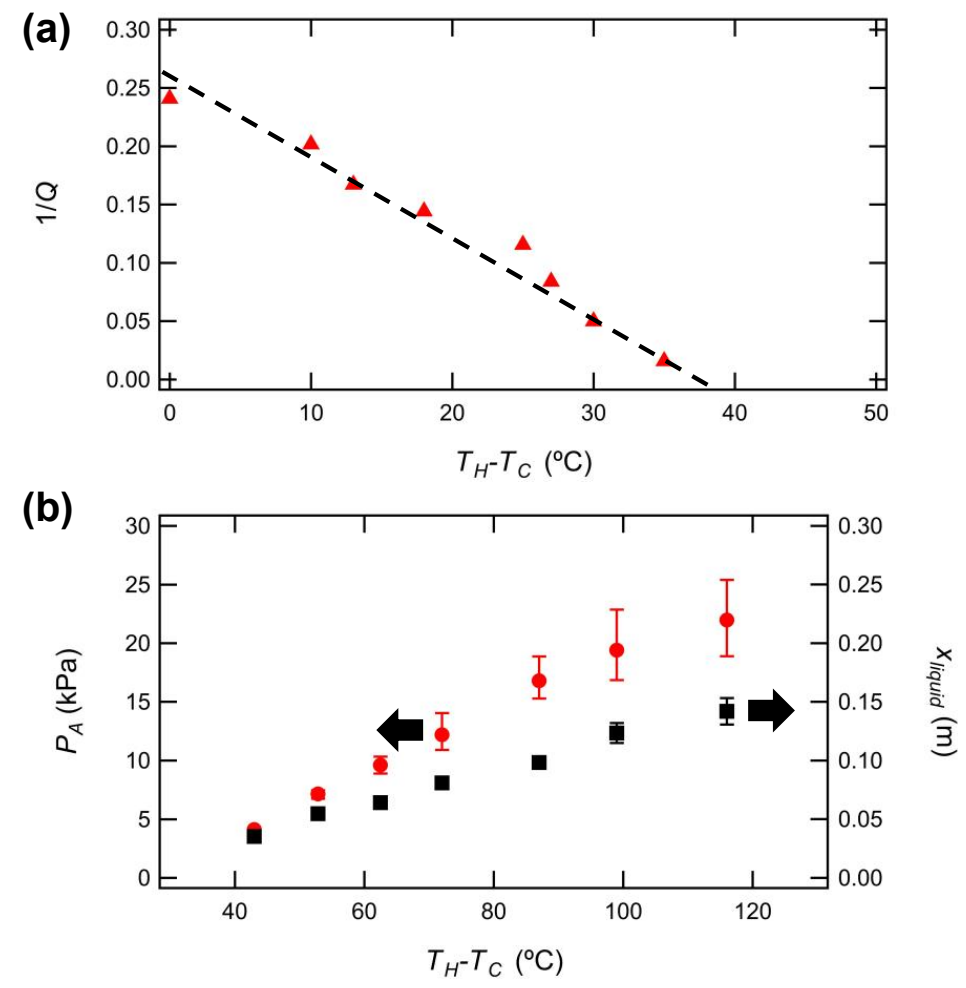

Figure 4. (a) $1 / Q$ and (b) pressure and displacement $X_{\text {liquid }}$ amplitude of liquid columns as a function of $T_{H}-T_{C}$. Dashed line is guide to the eye.

\section{Conclusion}

The design method of multi-cylinder type liquid piston Stirling engine (MCLPSE) was proposed to achieve target acoustic power generation. The experimental setup built based on the design optimization achieved the target acoustic power generation. 


\section{References}

[1] Yazaki, T., Iwata, A., Maekawa, T., and Tominaga, A., Applied Physics Letters, 81, 3128-3131 (1998).

[2] Ceperley, Peter H., The Journal of the Acoustical Society of America, 77, 1239-1244 (1985).

[3] Backhaus, S., and Swift, G. W. , Nature, 399, 335-338 (1999).

[4] West, C.D., British Patent No. 1507678 (1974).

[5] Martini, W.R., Proc., Intersoc. Energy Convers. Eng. Conf.; (United States) 2, (1983).

[6] Hyodo, H., Tamura, S., and Biwa, T., Journal of Applied Physics, 122, 114902 (2017).

[7] Murti, P., Takizawa, A., and Biwa, T., In Proceedings of the 27th International Congress on Sound and Vibration (ICSV 27), 11-16 July 2021.

[8] Biwa, T., Komatsu, R., and Yazaki, T., The Journal of the Acoustical Society of America, 129, 132-137 (2011).

[9] Murti, P., Hyodo, H. and Biwa, T., Journal of Applied Physics, 127, 154901 (2020).

[10] Biwa, T., Hasegawa, D., and Yazaki, T., Applied Physics Letters, 97, 034102 (2010).

[11] Tamura, S., Hyodo, H., and Biwa, T., Japanese Journal of Applied Physics, 58, 017001 (2018).

[12] Clark,J. P. and Ward,W. C. and Swift,G. W., The Journal of the Acoustical Society of America, 122, 3014 (2007).

[13] Yu, Z., Jaworski, J. A., Backhaus, S., Applied Energy, 99, 135-145 (2012). 